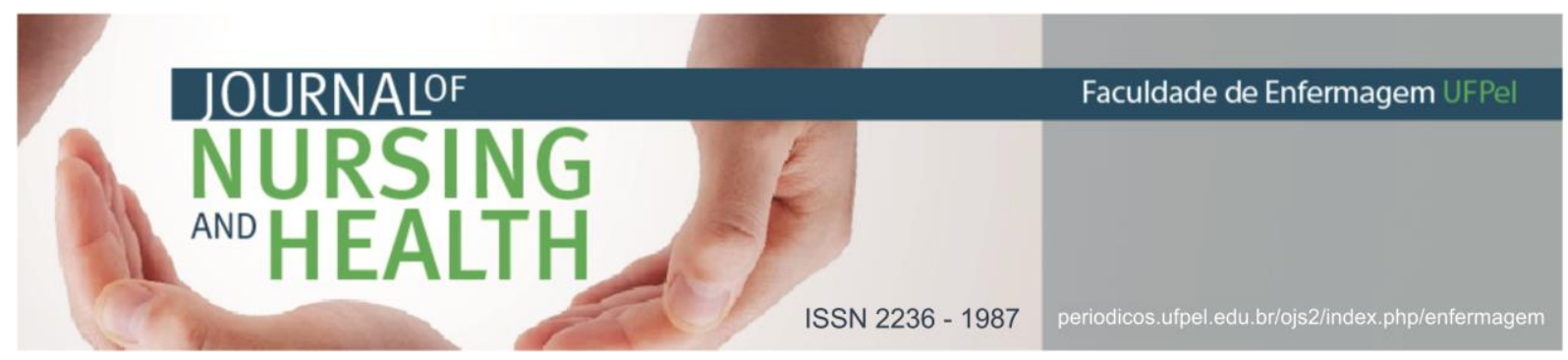

ARTIGO ORIGINAL

\title{
Perfil sociodemográfico e clínico de idosos em risco de quedas no sul do Brasil
}

\section{Sociodemographic and clinical profile of elderly at risk for falls in southern Brazil \\ Perfil sociodemográfico y clínico de ancianos en riesgo de caídas en el sur de Brasil}

Silva, Isadora Gabriella Paschoalotto¹; Peruzzo, Hellen Emilia²; Lino, Iven Giovana Trindade ${ }^{3}$; Marquete, Verônica Francisqueti ${ }^{4}$; Marcon, Sonia Silva ${ }^{5}$

Como citar este artigo: Silva IGP, Peruzzo HE, Lino IGT, Marquete VF, Marcon SS. Perfil sociodemográfico e clínico de idosos em risco de quedas no sul do Brasil. J. nurs. health. 2019;9(3):e199308

\section{RESUMO}

Objetivo: analisar o perfil sociodemográfico e clínico de idosos quanto ao risco de quedas em um município no Sul do Brasil. Métodos: estudo transversal realizado por meio de Vulnerable Elders Survey-13 e o Falls Risk Awareness Questionnaire, com 66 idosos recrutados por bola de neve, de janeiro a outubro de 2018. A análise estatística foi descritiva e inferencial. Resultados: houve 55 depoimentos de queda $(83,3 \%)$, tendo mais risco mulheres $(69,70 \%)$, com idade média de 75 anos $(45,5 \%)$. Hipertensão Arterial Sistêmica $(67,2 \%)$, problemas na coluna $(56,9 \%)$ e ansiedade $(53,4 \%)$ foram as morbidades mais frequentes e $51,5 \%$ fazem uso de duas ou mais medicações contínuas diariamente. Conclusão: risco de queda predominou em mulheres, com idade avançada, associada às condições de vulnerabilidade, histórico de quedas anteriores, uso de medicamentos contínuos e com pelo menos uma morbidade que afeta sua vida diária.

Descritores: Acidentes por quedas; Idoso; Vulnerabilidade em saúde.

\begin{abstract}
Objective: to analyze the sociodemographic and clinical profile of the elderly regarding the risk of falls in a municipality in southern Brazil. Methods: cross-sectional study conducted using Vulnerable Elders Survey-13 and the Falls Risk Awareness Questionnaire with 66 snowball-recruited elderly from January to October 2018. Statistical analysis was descriptive and inferential. Results: there were 55 testimonials of falls (83.3\%), with more risk women (69.7\%), with an average age of 75 years (45.5\%). Systemic Arterial Hypertension (67.2\%), back problems (56.9\%) and anxiety (53.4\%) were the most frequent morbidities and $51.5 \%$ used two or more continuous medications daily. Conclusion: risk of falling predominated in women, with advanced age, associated with vulnerability conditions, history of previous falls, use of continuous medications and at least one morbidity that affects their daily life.

Descriptors: Accidental falls; Aged; Health vulnerability.
\end{abstract}

1 Discente do curso de Enfermagem. Universidade Estadual de Maringá (UEM), Brasil. E-mail: isaagabriella@gmail.com http://orcid.org/0000-0003-2542-1488

2 Enfermeira. Doutora em Enfermagem. Universidade Estadual de Maringá (UEM), Brasil. E-mail: hellen_peruzzo@hotmail.com http://orcid.org/0000-0002-0786-0447

3 Enfermeira. Universidade Estadual de Maringá (UEM), Brasil. E-mail: iven_giovanna@hotmail.com http://orcid.org/0000-0003-0631-9971

4 Enfermeira. Universidade Estadual de Maringá (UEM), Brasil. E-mail: veronicafrancisqueti@hotmail.com http://orcid.org/0000-0002-8070-6091

5 Enfermeira. Doutora em Enfermagem. Universidade Estadual de Maringá (UEM), Brasil. E-mail: soniasilva.marcon@gmail.com https://orcid.org/0000-0002-6607-362X 


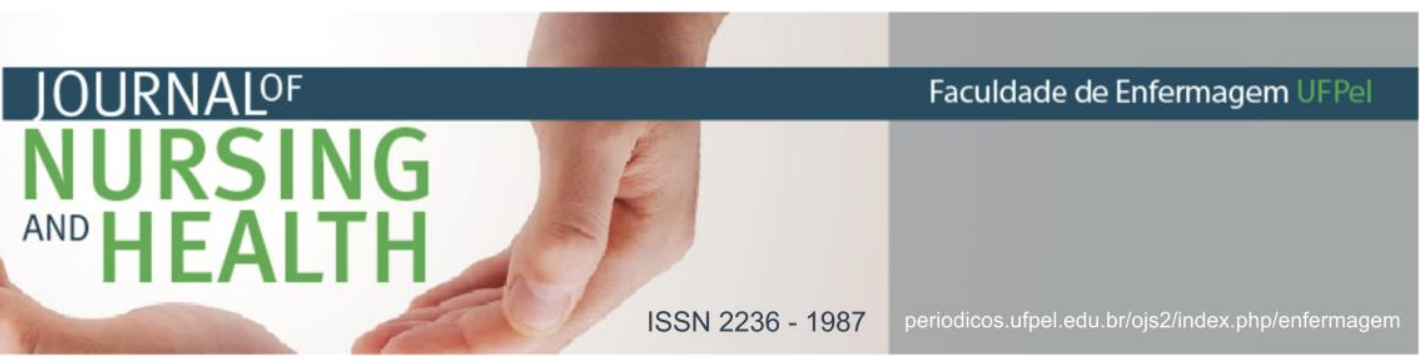

RESUMEN

Objetivo: analizar el perfil sociodemográfico y clínico de los adultos mayores en relación con el riesgo de caídas en un municipio del sur de Brasil. Métodos: estudio transversal realizado con la Encuesta de Ancianos Vulnerables-13 y el Cuestionario de Concientización sobre el Riesgo de Caídas con 66 ancianos reclutados con bolas de nieve de enero a octubre de 2018. El análisis estadístico fue descriptivo e inferencial. Resultados: hubo 55 testimonios de caídas (83.3\%), con mujeres de mayor riesgo (69.70\%), con una edad promedio de 75 años (45.5\%). La hipertensión arterial sistémica $(67,2 \%)$, problemas de espalda $(56,9 \%)$ y ansiedad $(53,4 \%)$ fueron las morbilidades más frecuentes y el 51,5\% usó dos o más medicamentos diariamente. Conclusión: el riesgo de caídas predominó en las mujeres, con edad avanzada, asociado con condiciones de vulnerabilidad, antecedentes de caídas previas, uso de medicamentos continuos y al menos una morbilidad que afecta su vida diaria.

Descriptores: Accidentes por caídas; Anciano; Vulnerabilidad en salud.

\section{INTRODUÇÃO}

Vários são os aspectos que trazem inquietação no cuidado a pessoa idosa. A transição demográfica no Brasil, que se iniciou em 1940 com a redução dos níveis de fecundidade e mortalidade, trouxe ao país envelhecimento da estrutura etária e aumento da expectativa de vida. 0 que antes era de 43,1 anos em 1940, passou para 75,2 em 2016. ${ }^{1}$ Neste contexto, cerca de $30 \%$ dos idosos sofre pelo menos uma queda ao ano, e ainda, que 15\% deles apresentam quedas recorrentes. ${ }^{2}$ Quando o idoso tem mais de 80 anos, os índices de duas ou mais quedas aumentam significativamente, se comparado aos idosos de 65 a 79 anos. ${ }^{2}$

Tendo em vista o crescente número de idosos, o risco para quedas passou a ser considerado um problema de saúde pública. Além da alta mortalidade decorrente da queda, destacam-se ainda outras consequências importantes como a restrição de mobilidade, incapacidade funcional, isolamento social, insegurança e medo, os quais agem num mecanismo cumulativo e em efeito dominó, com eventos prejudiciais à saúde e a qualidade de vida destes idosos. ${ }^{3}$
$\mathrm{Na}$ saúde, vulnerabilidade é entendida como a exposição do sujeito às situações que podem prejudicar sua condição de vida em um aspecto ou mais. ${ }^{4}$ A vulnerabilidade é expressa através do biológico/individual quando relacionado ao comportamento pessoal do indivíduo, autopercepção de saúde e limitações físicas/inabilidade na realização de atividades do cotidiano. ${ }^{5}$

0 envelhecimento quando somado à presença de Doenças Crônicas Não Transmissíveis interfere negativamente na autonomia do indivíduo, levando-o a dependência de cuidados. $^{6}$ Os pontos de vulnerabilidade individual, ou seja, a condição de saúde atual é referida como causa da queda pelas vítimas (principalmente nos casos de demência e diabetes mellitus). ${ }^{7}$

A queda é considerada um evento sinalizador do declínio da capacidade funcional em idosos, portanto, é importante avaliar os riscos e fatores que podem contribuir para que 0 evento aconteça. ${ }^{8} \mathrm{Na}$ busca por identificar mecanismos de enfrentamento e que possam minimizar os efeitos causais dos episódios de queda entre idosos, as 


\section{JOURNALOF \\ NURSING \\ AND HEALTH}

ISSN $2236-1987$

primeira casa após a igreja matriz. Neste, foi identificado a presença de dois idosos, sendo então realizado sorteio simples de qual dos dois seria incluído no estudo.

Ao término da entrevista foi solicitado ao idoso participante que indicasse um outro idoso residente em outro ou no mesmo bairro para ser convidado a participar do estudo. Isto foi feito até que as indicações começaram a se repetir sistematicamente. Ressalta-se que dentre as indicações feitas, nove idosos não aceitaram participar do estudo. Nestes casos, outros domicílios foram sorteados e definidos para iniciar um novo ciclo - sempre a primeira casa do lado esquerdo da quadra.

Durante a entrevista foi aplicado um questionário individual composto por questões relacionadas às características sociodemográficas com perguntas relacionadas aos aspectos sexo, idade, estado conjugal, escolaridade, ocupação atual e morbidades referidas pelos idosos juntamente com dois instrumentos validados, o Vulnerable Elders Survey13 (VES-13) e três sessões do Fall Risk Score.

O VES-13 foi criado em 2001, traduzido e adaptado transculturalmente para o português. ${ }^{10}$ $O$ instrumento é constituído por quatro itens e tem como objetivo avaliar a vulnerabilidade do idoso, pontuando a dificuldade em realizar Atividades Básicas da Vida Diária (ABVD) e Atividades Instrumentais da Vida Diária (AIVD). Essas atividades podem ser caracterizadas como agachar-se, realizar serviços domésticos e 


\section{JOURNAIOF

atividades cotidianas, como lidar com dinheiro, tomar banho sozinho e fazer compras, ou seja, avalia a dependência funcional do idoso para reconhecê-lo como frágil.

Já o Fall Risk Score foi elaborado por Downton, em 1993, traduzido e adaptado para português. ${ }^{11}$ Optou-se por utilizar apenas três sessões do instrumento neste estudo: a Avaliação Cognitiva Mini Exame do Estado Mental, que conta com questões para caracterização do estado mental do idoso, se ele se localiza no tempo e no espaço, se apresenta-se lúcido, com memória preservada e capacidade de acionar a linguagem; a Escala de Risco de Quedas, que aborda questões relacionadas ao estado de saúde, presença ou não de morbidade que afetam a vida diária, uso de medicações contínuas e déficits sensitivos ou na marcha (modo de andar); e por fim a sessão Quedas, que apresenta questões sobre episódios de quedas anteriores, a frequência com que elas aconteceram nos últimos 12 meses, caracterização da queda quanto sua causa, local e suas consequências.

Os dados foram organizados em uma planilha do programa Excel 2013 ${ }^{\circledR}$, e posteriormente analisados com auxílio da estatística descritiva e inferencial, cruzando as informações de sexo, idade, vulnerabilidade, morbidades autorreferidas e uso de medicamentos diários, histórico de queda com o índice de risco de queda que os entrevistados apresentaram. Para tanto foi utilizando o teste exato de Fischer no software $R$ versão 3.5.1, adequado aos valores esperados (menores que cinco), com significância estatística de $5 \%$.

No desenvolvimento do estudo foram respeitados os preceitos éticos disciplinados pela Resolução $\mathrm{n}^{\circ}$ 466/2012 do Conselho Nacional de Saúde e seu projeto foi aprovado pelo Comitê Permanente de Ética em Pesquisas com Seres Humanos da Universidade Estadual de Maringá (Certificado de apresentação para Apreciação Ética: 89100618.9.0000.0104). Todos os participantes assinaram o Termo de Consentimento Livre e Esclarecido, em duas vias.

\section{RESULTADOS}

$\mathrm{Na}$ Tabela 1 consta as variáveis sociodemográficas associadas ao risco de queda. Os 66 idosos participantes tinham idade média de 75 anos (amplitude de 60 a 90 anos), 69,7\% eram do sexo feminino, $83,3 \%$ já havia sofrido quedas e $45,5 \%$ julgavam seu estado de saúde como bom. A morbidade mais comum informada foi a Hipertensão Arterial Sistêmica (HAS), presente em $67,2 \%$ dos idosos, seguido por problemas na coluna $(56,9 \%)$ e ansiedade $(53,4 \%)$. Pouco mais da metade $(51,5 \%)$ faz uso de duas ou mais medicações contínuas diariamente, $34,5 \%$ relatou dificuldades nas AIVD e $15,5 \%$ na realização de ABVD.

Não foram encontradas diferenças significativas que associem as variáveis autopercepção de saúde, dificuldades na realização de atividades diárias e a vulnerabilidade dos idosos com os episódios de queda anteriores (Tabela 2). Cerca de 87,5\% os idosos que avaliaram sua saúde como regular ou ruim já haviam sofrido 
AWHEA

queda anteriormente. Tanto para as AIVDs, como para as ABVDs, a maioria dos participantes já haviam tido episódios de queda anteriormente (84,0\% e $90,9 \%$, respectivamente). 0 histórico de quedas anteriores também foi maior entre os indivíduos que apresentavam estado de vulnerabilidade $(97,0 \%)$.

Constatou-se que a maioria dos idosos haviam sofrido pelo menos um episódio de queda anteriormente $(83,3 \%)$ e mais da metade das quedas,
ISSN 2236 - 1987

(56\%) teriam ocorrido nos 12 meses precedentes à entrevista.

Quanto às características das quedas, a maioria foi relatada decorrente da própria altura $(54,5 \%)$, sendo no pátio da casa $(20 \%)$, sem consequências negativas $(38,2 \%)$ ou apenas com escoriações $(27,3 \%)$. Os idosos que necessitaram de hospitalização pós-queda contabilizam $12,7 \%$, das quais $28,6 \%$ dos casos resultaram em procedimentos cirúrgicos para correção de fraturas.

Tabela 1: Variáveis associadas ao risco de quedas em idosos do município de Mandaguari - PR, Brasil, 2018. $n=66$

\begin{tabular}{|c|c|c|c|c|c|c|}
\hline \multirow[t]{3}{*}{ Variáveis } & & \multicolumn{5}{|c|}{ Risco para queda } \\
\hline & & \multicolumn{2}{|l|}{ Sim } & \multicolumn{2}{|c|}{ Não } & \multirow[t]{2}{*}{ p-valor* } \\
\hline & & $\mathrm{n}$ & $\%$ & $\mathrm{~N}$ & $\%$ & \\
\hline \multirow[t]{3}{*}{ Sexo } & Feminino & 24 & 52,1 & 22 & 47,8 & \multirow{3}{*}{0,5117} \\
\hline & Masculino & 10 & 50,0 & 10 & 50,0 & \\
\hline & $60-69$ & 7 & 26,1 & 17 & 70,8 & \\
\hline \multirow[t]{2}{*}{ Idade (em anos) } & $70-79$ & 16 & 59,2 & 11 & 40,7 & \multirow[t]{2}{*}{0,2237} \\
\hline & $>80$ & 11 & 73,3 & 4 & 26,6 & \\
\hline \multirow{3}{*}{ Vulnerabilidade } & Sim & 15 & 75,0 & 5 & 25,0 & \multirow{3}{*}{0,0885} \\
\hline & Não & 19 & 41,3 & 27 & 58,7 & \\
\hline & Nenhuma & 0 & 0,0 & 13 & 100,0 & \\
\hline \multirow[t]{2}{*}{ Medicações diárias } & Toma um & 5 & 26,3 & 14 & 73,6 & \multirow[t]{2}{*}{$<0,001$} \\
\hline & Toma dois ou mais & 29 & 85,2 & 5 & 14,7 & \\
\hline \multirow{3}{*}{ Quedas anteriores } & Sim & 33 & 60,0 & 22 & 40,0 & \multirow{2}{*}{0,2062} \\
\hline & Não & 1 & 9,0 & 10 & 90,9 & \\
\hline & Interferem na vida & 34 & 53,1 & 30 & 46,8 & \multirow{3}{*}{0,0364} \\
\hline \multirow{2}{*}{$\begin{array}{l}\text { Morbidades (HAS, } \\
\text { DM, insônia, etc.) }\end{array}$} & Não interferem na & & & & & \\
\hline & vida & 0 & 0,0 & 2 & 100,0 & \\
\hline
\end{tabular}

Fonte: dados da pesquisa, 2018.

Entre os participantes, 24,2\% foram classificados como vulneráveis. Desses, $60 \%$ já haviam apresentado pelo menos um episódio de queda nos últimos 12 meses. Observou-se que o risco para queda aumenta à medida que a pessoa envelhece, posto que
$73,3 \%$ dos idosos com 80 anos ou mais apresentam maior risco.

Apesar de não apresentar diferença significativa, pessoas do sexo feminino $(52,1 \%)$, em estado de vulnerabilidade $(75,0 \%)$, com histórico de queda anterior $(60,0 \%)$ e 
diagnóstico de morbidades que afetavam a vida diária $(53,1 \%)$ tiveram mais chance de apresentar novos episódios de queda. Quanto a variável medicações diárias, 85,29\% indivíduos que faziam uso de duas ou mais medicações contínuas, apresentaram risco para queda, com diferença significativa quando comparados às pessoas que não faziam uso de medicamentos ou usavam apenas um ( $p$-valor $<0,001)$.

Tabela 2 - Variáveis associadas ao histórico de quedas em idosos do município de Mandaguari - PR, Brasil, 2018. $\mathrm{n}=66$

\begin{tabular}{|c|c|c|c|c|c|c|c|}
\hline \multirow{3}{*}{ Variáveis } & & & \multicolumn{4}{|c|}{ Quedas anteriores } & \multirow[t]{3}{*}{ p- valor* } \\
\hline & & & Sim & & Nãc & & \\
\hline & & & $\mathrm{n}$ & $\%$ & $\mathrm{n}$ & $\%$ & \\
\hline \multirow{4}{*}{$\begin{array}{l}\text { Autopercepção } \\
\text { saúde } \\
\text { Dependência } \\
\text { realização } \\
\text { atividades } \\
\text { cotidiano }\end{array}$} & \multirow{2}{*}{$\mathrm{da}$} & Boa/Muito boa & 41 & 82,0 & 9 & 18,0 & \multirow[t]{2}{*}{1} \\
\hline & & Regular/Ruim & 14 & 87,5 & 2 & 12,5 & \\
\hline & \multirow{2}{*}{$\begin{array}{r}\text { para } \\
\text { de } \\
\text { do }\end{array}$} & AIVD & 21 & 84,0 & 4 & 16,0 & 1 \\
\hline & & ABVD & 10 & 90,9 & 1 & 9,1 & 0,6744 \\
\hline Vulnerabilidade & & $\begin{array}{l}\text { Sim } \\
\text { Não }\end{array}$ & $\begin{array}{l}33 \\
22\end{array}$ & $\begin{array}{l}97,0 \\
68,7\end{array}$ & $\begin{array}{l}1 \\
10\end{array}$ & $\begin{array}{l}2,9 \\
31,2\end{array}$ & 1 \\
\hline
\end{tabular}

Fonte: dados da pesquisa, 2018.

\section{DISCUSSÃO}

0 risco de queda entre idosos é frequente, um estudo realizado em uma pesquisa multicêntrica em Barueri, São Paulo e Cuiabá, Mato Grosso do Sul no período de 2009 a 2010, 38,6\% dos idosos entrevistados já haviam apresentado queda anteriormente ${ }^{2}$ e tem características diferentes. ${ }^{2}$ Assim como no presente estudo, em pesquisa realizada na Bahia, junto à 22 participantes de ambos os sexos, com o objetivo de avaliar a propensão à queda entre idosos cadastrados em uma UBS e os fatores de risco associados, a partir da investigação de aspectos sociodemográficos, uso de medicamentos, patologias autor referidas, prática de atividades físicas e dados antropométricos, este estudo apresentou feminização da velhice, já que a população feminina em idosos é maior que a masculina. ${ }^{7}$ Esta constatação pode ser explicada em decorrência da maior expectativa de vida feminina, fundamentada pela menor exposição aos fatores de risco de mortalidade por causas externas, além de uma postura diferente frente ao autocuidado. ${ }^{12}$ Porém, assim como neste estudo, as mulheres possuem os maiores riscos à queda. ${ }^{2-3,7}$ Estudo entre idosos institucionalizados apontou que o sexo feminino teve risco 3,9 vezes maior de queda, quando comparado ao sexo masculino. ${ }^{13}$

Quanto às morbidades, todos os participantes apresentaram pelo menos uma, sendo HAS relatada em outros estudos ${ }^{3}$ considerada uma doença frequente do processo de envelhecimento. ${ }^{7}$ A HAS está relacionada à diminuição da qualidade de vida idosos, e assim como a depressão também foi relatada em estudos com idosos com risco de quedas. ${ }^{2}$ Em estudo com o objetivo de explorar as características da última queda em idosos brasileiros, realizado 


\section{JOURNALOF \\ NURSING \\ AND HEALTH}

ISSN $2236-1987$

psicotrópicos, medicamentos

com 774 idosos nos municípios de Barueri, São Paulo e Cuiabá, Mato Grosso do Sul entre os anos de 2008 e 2009 identificou que 43,1\% dos idosos que caíram duas vezes ou mais apresentavam-se depressivos. 2 Estes fatores, quando somado ao declínio da capacidade funcional, que acontece naturalmente no processo de envelhecer, tem como consequência a perda da autonomia deste idoso. ${ }^{13}$

A maioria dos idosos em estudo fazem uso de pelo menos um medicamento continuamente, resultado semelhante ao encontrado em outros estudos. ${ }^{2-3,7,14}$ Sabe-se que o uso de agentes farmacêuticos e a presença de doenças vasculares são condições associadas que aumentam a suscetibilidade à queda. ${ }^{15}$ No presente estudo, a associação destas variáveis com o risco de queda evidenciou que grande parte dos idosos que fazem uso de duas ou mais medicações diárias possuem maior risco para queda, risco presente também em mais da metade dos que apresentam uma ou mais morbidade que interferem na vida diária.

Os medicamentos com maior consumo entre os entrevistados foram hipoglicemiantes (p. ex. Metformina), diuréticos ( $p$. ex. Furosemida), inibidores da enzima conversora de angiotensina (p. ex. Captopril) e antagonistas do receptor da angiotensina II (p. ex. Losartana), além de medicamentos hipnotizantes para a indução do sono. O Instituto para Práticas Seguras no Uso de Medicamentos (ISMP) do Canadá, em uma pesquisa, constatou que as classes de medicamentos mais associadas à ocorrência de queda são: opioides, utilizados no tratamento de doenças cardiovasculares e hipoglicemiantes (incluindo a insulina). Além disso foi observado que o uso de diurético está associado às quedas pois causa sintomas no paciente como a poliúria e possivelmente a nictúria. ${ }^{16}$

O ISMP em 2017 divulgou recomendações aos profissionais de saúde para reduzir o risco de quedas associadas a medicamentos: revisar a prescrição médica; atentar-se nas admissões, transferências e alta hospitalar onde o cuidado é transferido; conforme necessidade, realizar adequações na prescrição de medicações com potencial risco de queda; instruir o paciente e a família sobre os medicamentos e os respectivos sintomas que podem levar a queda; avaliar 0 uso dos medicamentos e as interações medicamentosas que causam hipoglicemia (principal causa de queda). ${ }^{16}$ Ressalta-se a importância de seguir essas recomendações pois, o uso crônico de medicamentos podem potencializar o risco de queda.

Neste estudo, a prevalência de quedas entre os idosos entrevistados foi de $83,3 \%$, percentual maior do que - observado em uma pesquisa realizada em Natal, Rio Grande do Norte, em 2012, onde 53,6\% dos idosos pertencentes a uma UBS da cidade já haviam sofrido pelo menos um episódio de queda, sendo excluídos da pesquisa idosos dependentes de cuidados nas ABVD ou que possuíssem alguma enfermidade agravante. ${ }^{17} \mathrm{E}$ do estudo que determinou a prevalência de quedas em idosos vinculados a uma UBS de Goiânia ${ }^{3}$ e Belo Horizonte. ${ }^{2}$ 


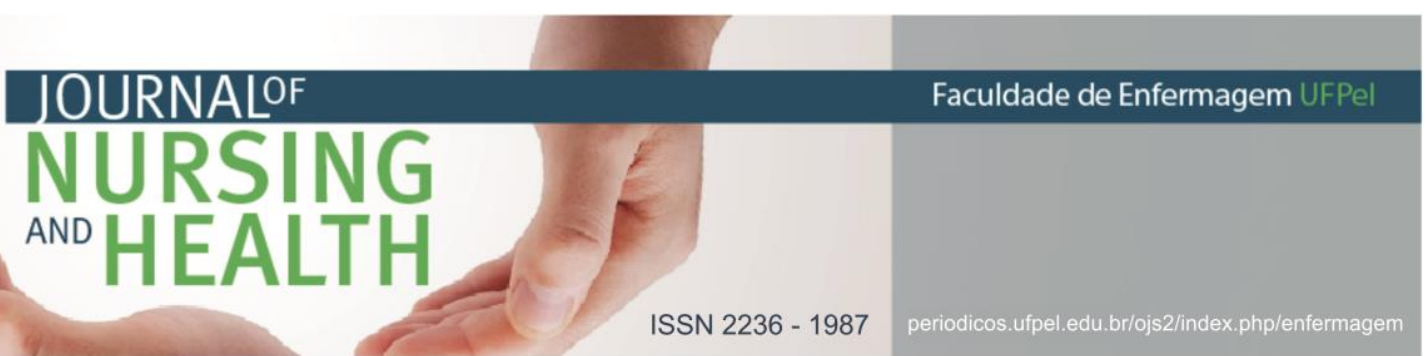

O histórico de quedas e a presença de vulnerabilidade, ${ }^{18}$ apontou a dificuldade dos idosos para realizar AIVD e ABVD, ${ }^{19}$ isso representa que o impacto do episódio de queda é tão significativo que quase $50 \%$ dos idosos passaram a ter dificuldades ou deixaram de realizar a atividade, portanto, a queda na velhice pode acarretar prejuízos na realização das atividades diárias e declínios na saúde do idoso. ${ }^{17}$ Concomitantemente, a perda da independência deste idoso no pós-queda se dá pela presença de dores e incapacidades, medo de uma nova queda e atitudes protetoras de familiares/cuidadores. ${ }^{20}$ Além disso, também há consequências como os prejuízos financeiros, hospitalizações, danos emocionais e psicológicos. ${ }^{20}$

Foi observado que com o aumento da idade a porcentagem de risco de queda também aumenta significativamente. As alterações progressivas presentes no processo de envelhecimento como perda muscular, dificuldade em manter a estabilidade postural lentificação da marcha e perda de equilíbrio, ${ }^{21}$ perda de força de preensão manual e de audição, fatores que predispõem o indivíduo à queda. ${ }^{22}$ A faixa etária com maior predominância de queda foi 80 anos ou mais, resultado semelhante ao encontrado em outro estudo realizado com 127 idosos hospitalizados por queda em um município de pequeno porte na Bahia em 2015. ${ }^{22}$

A autopercepção de saúde como regular e ruim se assemelha ao encontrado em um estudo realizado no Distrito Federal, Brasil no período de 2012 a 2015 , onde $47,7 \%$ dos idosos consideraram sua saúde como regular ou ruim. ${ }^{18}$ Tal variável se associa à prevalência de quedas, assim como encontrado em outros estudos realizados na Bahia ${ }^{7}$ e em Brasília. A ocorrência de queda em idosos pode levar a dificuldades na realização das atividades de vida diárias, ${ }^{20}$ decorrentes da incapacidade, como por exemplo, no caso da presença de fraturas, perda visual como constatado em um estudo realizado com 24 idosos em um centro de saúde de Fortaleza, Ceará no ano de 2003 , onde $95,8 \%$ dos idosos que apresentaram risco de queda possuíam dificuldades visuais ${ }^{23}$ ou pelo medo de cair novamente, que se fez presente em $60 \%$ dos participantes de uma pesquisa com 193 idosos institucionalizados no município de Porto Alegre, Rio Grande do Sul, Brasil em 2016. ${ }^{24}$ Levando a um ciclo que se repete, uma vez que o idoso que apresenta limitações para a realização de atividades de vida diária possui maior risco para novos de episódio de queda, estudo realizado em Pelotas, Rio Grande do Sul, associou a ocorrência de quedas à capacidade funcional, idosos dependentes para atividades de vida diária apresentaram maior prevalência de quedas quando comparado aos idosos independentes $(\mathrm{RP}=1,38 ; \mathrm{IC} 95 \% 1,10-1,48) .{ }^{25}$

0 enfermeiro tem papel fundamental na avaliação do idoso, principalmente no que diz respeito à conservação da saúde, funcionalidade e cognição dos idosos, tendo como objetivo minimizar o risco de quedas. Ao conhecer as mudanças na mobilidade e funções orgânicas da maior idade, a enfermagem pode atuar estimulando a alimentação, prática de exercícios e um ambiente seguro. ${ }^{26} \mathrm{~A}$ Consulta de Enfermagem é 


\section{ISSN 2236 - 1987}

instrumento que torna isso possível, instituindo uma relação de ajuda onde o profissional enfermeiro, interagindo com o paciente, atua na promoção de saúde, prevenção de doenças e limitação dos danos possíveis.

0 enfermeiro tem a competência para promover intervenções preventivas que vão evitar maiores incapacidades, complicações secundárias e garantir qualidade de vida e dignidade ao idoso, mesmo após a queda. ${ }^{27}$ Principalmente na Atenção Primária à Saúde, onde os objetivos ao atender idosos são ampliar a longevidade, identificar fragilidade e intervir em prevenção e reversão. ${ }^{28}$

A dependência e a vulnerabilidade são observadas em idosos com mais de 85 anos, ou mais jovens quando apresentam limitações ou doenças que reduzam sua autonomia. Neste contexto, os cuidadores familiares têm importância ímpar, embora a dependência do idoso altere a rotina e funcionamento da família, esta desempenha papel importante no cuidado e recuperação do idoso após a queda. Nesse momento, os membros familiares podem oferecer recursos físicos, emocionais e um sistema de apoio necessário. Além disso, o cuidado familiar proporciona realização pessoal aos idosos, sentimento de inclusão social, podendo também promover a reestruturação dos laços familiares. ${ }^{29}$

\section{CONCLUSÃO}

Os resultados permitiram analisar o perfil e a vulnerabilidade de idosos com risco para queda, na maioria do sexo feminino, caracterizados como vulneráveis, com histórico de quedas anteriores, idade avançada, faziam uso de medicamentos contínuos e possuíam pelo menos uma morbidade que afetou sua vida diária.

Identificando o grupo de maior risco, a causa da queda e suas consequências, o enfermeiro e os demais profissionais de saúde da equipe de saúde da família podem elaborar estratégias mais assertivas, garantindo um cuidado personalizado à população, qualificando a assistência e diminuindo sua ocorrência. Destarte, o estudo fortalece a importância da identificação de episódios de queda, em virtude principalmente do impacto que trazem a qualidade de vida destes idosos, já que podem contribuir para incapacidades, vulnerabilidade e perda da independência. Neste sentido, futuros estudos são necessários para avanços no planejamento para a prevenção desses eventos.

Como limitações, o uso de medicamentos e as morbidades associadas são fatores que podem ter contribuído para a queda, contudo a perda de memória referente à ocorrência do número de quedas pode não corresponder exatamente a realidade dos idosos.

\section{REFERÊNCIAS}

1 Myrrha LJD, Turra CM, Wajnman S. A contribuição dos nascimentos e óbitos para o envelhecimento populacional no Brasil, 1950 a 2100. Revista latinoamericana de población [Internet]. 2017 jan/jun[citado em 2019 nov 18];11(20):37-54. Disponível em: https://revistarelap.org/index.php/re lap/article/view/33 


\section{JOURNALOF \\ NURSING \\ ANO HEALTH}

ISSN 2236 - 1987

18];22(6):2063-71. Disponível em: http://www.scielo.br/pdf/csc/v22n6/ 1413-8123-csc-22-06-2063.pdf

7 Luzardo AR. Vulnerabilidade, fatores preditores e repercussões da queda para idosos hospitalizados [tese]. Florianópolis (SC): Universidade Federal de Santa Catarina; 2015.

8 Souza LHR, Brandão JCS, Fernandes AKC, Cardoso BLC. Queda em idosos e fatores de risco associados. Revista de atenção à saúde [Internet]. 2017 out/dez[citado em 2019 ago 18];15(54):55-60. Disponível em: http://seer.uscs.edu.br/index.php/re vista_ciencias_saude/article/downloa d/4804/pdf

9 Instituto Brasileiro de Geografia e Estatística (IBGE). Cidades: Mandaguari (PR) [Internet]. [acesso em 2019 nov 19]. Disponível em: https: / / www.ibge.gov.br/cidades-eestados/pr/mandaguari.html

10 Maia FOM, Duarte YAO, Secoli SR, Santos JLF, Lebrão ML. Adaptação transcultural do Vulnerable Elders Survey -13 (VES-13): contribuindo para a identificação de idosos vulneráveis. Rev. Esc. Enferm. USP. [Internet]. 2012[citado 2019 ago 18];46(n.esp.):116-22. Disponível em: http:/ / www.scielo.br/pdf/reeusp/v46 nspe/17.pdf

11 Schiaveto FV. Avaliação do risco de quedas em idosos na comunidade [dissertação] [Internet]. Ribeirão Preto (SP): Universidade de São Paulo; 2008[acesso em 2019 nov 19]. Disponível em: https: / / teses.usp.br/teses/disponivei s/22/22132/tde-19122008153736/publico/FabioVeigaSchiaveto. pdf 


\section{JOURNALOF \\ NURSING \\ ANO HEALTH}

12 Borges JES, Camelier AA, Oliveira LVF, Brandão GS. Qualidade de vida de idosos hipertensos e diabéticos da comunidade: um estudo observacional. Rev. Pesqui. Fisioter. [Internet]. 2019[citado em 2019 ago 18];9(1):7484. Disponível em: https: / /www5.bahiana.edu.br/index. $\mathrm{php} /$ fisioterapia/article/download/22 $49 / 2293$

13 Silva JMN, Barbosa MFS, Castro POCN, Noronha MM. Correlação entre o risco de queda e autonomia funcional em idosos institucionalizados. Rev. bras. geriatra. gerontol. (Online) [Internet]. $2013 \mathrm{abr} /$ jun[citado em 2019 nov 18];16(2):337-46. Disponível em:

http://www.scielo.br/pdf/rbgg/v16n2 /13.pdf

14 Dutra AP, Porto SA, Reis LA, Morais KCS, Lima LS, Santos KT. Risco de queda e uso de medicamento em idosos. Rev. Pesqui. Fisioter. [Internet]. 2017[citado em 2019 ago 18];7(4):498-503. Disponível em: https: //www5.bahiana.edu.br/index. $\mathrm{php} /$ fisioterapia/article/view/1587/1 043

15 Barros, ALNL (org). Diagnósticos de enfermagem da Nanda-l: definições e classificação 2018-2020 [Internet]. 11 ${ }^{\text {a }}$ ed. Porto Alegre: Artmed; 2018[acesso em 2019 nov 19]. Disponível em: http: / /www.nascecme.com.br/2014/ wp-content/uploads/2018/08/NANDAI-2018_2020.pdf

16 Instituto de Práticas Seguras no Uso de Medicamentos (ISMP Brasil). Boletim ISMP: medicamentos associados à ocorrência de quedas [Internet]. 2017 fev[acesso em 2019 nov 19]6(1):1-6. Disponível em:
ISSN $2236-1987$

https: / /www.ismp-brasil.org/site/wpcontent/uploads/2017/02/IS_0001_17 _Boletim_Fevereiro_ISMP_210x276mm .pdf

17 Santos RKM, Maciel ACC, Britto HMJS, Lima JCC, Souza TO. Prevalência e fatores associados ao risco de quedas em idosos adscritos a uma Unidade Básica de Saúde do município de Natal, RN, Brasil. Ciênc. Saúde Colet. [Internet]. 2015[citado em 2019 ago 18];20(12):3753-62. Disponível em: http: / /www.scielo.br/pdf/csc/v20n12 /1413-8123-csc-20-12-3753.pdf

18 Amancio TG, Oliveira MLC, Amancio VS. Factors influencing the condition of vulnerability among the elderly. Rev. bras. geriatra. gerontol. (Online) [internet]. 2019 Aug[cited 2019 Nov 09];22(2):e180159. Available from: http://www.scielo.br/pdf/rbgg/v22n2 /1809-9823-rbgg-22-02-e180159.pdf

19 Alves RLT, Silva CFM, Pimentel LN, Costa IA, Souza ACS, Coelho LAF. Evaluation of risk factors that contribute to falls among the elderly. Rev. bras. geriatra. gerontol. (Online) [Internet]. 2017[cited em 2019 Nov 18];20(1):56-66. Available from: http://www.scielo.br/pdf/rbgg/v20n1 /1809-9823-rbgg-20-01-00056.pdf

20 Lima RJ, Pimenta CJL, Bezerra TA, Viana LRC, Ferreira GRS, Costa KNFM. Capacidade funcional e o risco de quedas em pessoas idosas. Rev Rene (Online) [Internet]. 2017 set/out[citado em 2019 ago 18];18(5):616-22. Disponível em: http: / / www.periodicos.ufc.br/rene/a rticle/download/30823/71486

21 Esquenazi D, Silva SRB, Guimarães MAM. Aspectos fisiopatológicos do envelhecimento humano e quedas em 


\section{NURSING \\ AND \\ HEALTH}

idosos. Revista hospital universitário Pedro Ernesto [Internet]. 2014 abr/jun[citado em 2019 ago 18];13(2):11-20. Disponível em: http://revista.hupe.uerj.br/audiencia _pdf .asp?aid2=467\&nomeArquivo=v13n 2a03.pdf

22 Peixoto TM, Artelosa RCC, Silva LAT, Santos TSM. Causas e consequências de quedas em idosos atendidos no hospital de Santo Estevão, BA. Rev. biocienc. [Internet]. 2015[citado em 2019 ago 18];21(2):93-100. Disponível em: http://periodicos.unitau.br/ojs/index .php/biociencias/article/download/18 83/1537

23 Machado TR, Oliveira CJ, Costa FBC. Avaliação da presença de risco para queda em idosos. Rev. eletrônica enferm. [Internet]. 2009 [citado em 2019 nov 09];11(1):32-8. Disponível em:

http://www.fen.ufg.br/revista/v11/n 1/v11n1a04.htm

24 Rosa VPP, Cappellari FCBD, Urbanetto JS. Analysis of risk factors for falls among institutionalized elderly persons. Rev. bras. geriatra. gerontol. (Online) [Internet]. 2019[cited 2019 Nov 09];22(1):e180138. Available from: http://www.scielo.br/pdf/rbgg/v22n1 /1809-9823-rbgg-22-01-e180138.pdf

25 Vieiria LS, Gomes AP, Bierhals IO, Farías-Antúnez S, Ribeiro CG, Miranda VIA, et al. Quedas em idosos no Sul do Brasil: prevalência e determinantes. Rev. saúde pública (Online) [Internet]. 2018[citado em 2019 ago 18];52(22):1 $13 . \quad$ Disponível em: http: / / www.scielo.br/pdf/rsp/v52/pt _0034-8910-rsp-S1518-

87872018052000103.pdf
ISSN 2236 - 198

26 Costa JC, Lima MO, Pequeno JA, Lima MO, Gomes MMS, Junior AAL. Trauma por queda em idosos e a assistência de enfermagem. Ver. Saúde. [Internet]. 2017[citado em 2019 ago 18];11(1):40. Disponível em: http://revistas.ung.br/index.php/sau $\mathrm{de} /$ article/view/3126/2263

27 Fernandes AIC. Fatores de risco de queda num centro de reabilitação: contributos da enfermagem em reabilitação [dissertação] [Internet]. Évora (PT): Universidade de Évora; 2017[acesso em 2019 nov 19]. Disponível em: https://repositorio.ipbeja.pt/bitstrea m/20.500.12207/4553/1/Ana\%20Ferna ndes.pdf

28 Cabral R, Dellaroza MSG, Carvalho BG, Zani AV. Elderly care in primary health care from the perspective of health professionals. Ciênc. cuid. saúde. [Internet]. 2019[citado em 2019 jun 10];18(2). Disponível em: http://periodicos.uem.br/ojs/index.p $\mathrm{hp} /$ CiencCuidSaude/article/view/4502 6/pdf_1

29 Pereira-Llano PM, Santos F, Rodrigues MCT, Lemões MAM, Lange C, Santos SSC. The family in the care process of an elderly after a fall accident. Rev. Pesqui. (Univ. Fed. Estado Rio J., Online). [Internet]. 2016 jul/set[cited em 2019 Aug 18];8(3):4717-24. Available from: http://www.seer.unirio.br/index.php /cuidadofundamental/article/downlo $\mathrm{ad} / 2973 / \mathrm{pdf}$

Data de submissão: 07/08/2019

Data de aceite: 18/11/2019

Data de publicação: 24/11/2019 